\title{
Few-Shot Text Generation with Natural Language Instructions
}

\author{
Timo Schick and Hinrich Schütze \\ Center for Information and Language Processing, LMU Munich, Germany \\ schickt@cis. lmu.de
}

\begin{abstract}
Providing pretrained language models with simple task descriptions in natural language enables them to solve some tasks in a fully unsupervised fashion. Moreover, when combined with regular learning from examples, this idea yields impressive few-shot results for a wide range of text classification tasks. It is also a promising direction to improve data efficiency in generative settings, but there are several challenges to using a combination of task descriptions and example-based learning for text generation. In particular, it is crucial to find task descriptions that are easy to understand for the pretrained model and to ensure that it actually makes good use of them; furthermore, effective measures against overfitting have to be implemented. In this paper, we show how these challenges can be tackled: We introduce GENPET, a method for text generation that is based on pattern-exploiting training, a recent approach for combining textual instructions with supervised learning that only works for classification tasks. On several summarization and headline generation datasets, GENPET gives consistent improvements over strong baselines in few-shot settings. ${ }^{1}$
\end{abstract}

\section{Introduction}

Pretraining large neural networks with a language modeling objective has led to significant improvements throughout NLP (Peters et al., 2018; Howard and Ruder, 2018; Radford et al., 2018; Devlin et al., 2019; Raffel et al., 2020; Brown et al., 2020, i.a.). Further improvements are often possible by choosing a different pretraining objective that more closely matches the downstream task of interest. Examples include casing prediction for named entity recognition (Mayhew et al., 2020), gap sentence generation for summarization (Zhang et al.,

\footnotetext{
${ }^{1}$ Our implementation of GENPET and code to recreate our few-shot training datasets is publicly available at https: //github.com/timoschick/pet.
}

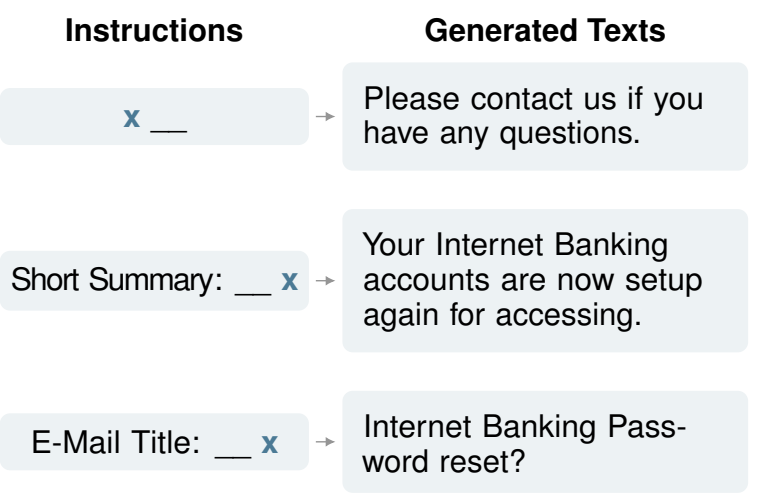

Figure 1: Texts generated by PEgasus-large with different instructions for input $\mathbf{X}=$ Dear John, Your Internet Banking accounts are now setup again for accessing. The login id is still your main account with the password being reset to the last six (6) digits of your SSN. Without any instructions, the model simply generates a continuation of the given input (top). Providing an instruction makes it generate an appropriate summary (center) or e-mail title (bottom) even in zero-shot settings and enables much more data-efficient learning.

2020), and sentence unshuffling for discourse representations (Lee et al., 2020).

While such approaches can significantly reduce the amount of training data required, they typically still do not perform well if only a handful of examples is available for the downstream task, which is a common scenario for many real-word uses of NLP. In such few-shot settings, however, significant gains are possible by reversing what is adapted to what: Instead of making pretraining more similar to a downstream task, we can reformulate the downstream task to make it more similar to the pretraining objective. For masked language models (e.g., Devlin et al., 2019; Lewis et al., 2020), one such reformulation technique is to convert inputs to cloze questions by adding a text snippet that contains some form of task description, often in the form of a short prompt (Radford et al., 2019; Schick and Schütze, 2021a). Besides making pretraining and finetuning more similar, this approach 
has the compelling benefit of enabling users to $e x$ plain a task to a pretrained model, making it much easier for the model to understand the task. This is illustrated in Figure 1, where a pretrained language model is given the same input with different instructions and adapts its output accordingly.

The idea of providing task descriptions even works in an unsupervised setting (Radford et al., 2019) or when examples are simply provided as additional context (Brown et al., 2020); however, it only unfolds its full potential when combined with gradient-based training on a handful of labeled examples (Schick and Schütze, 2021b). Unfortunately, current approaches for doing so are limited to text classification tasks (Schick and Schütze, 2021a). Inspired by their success, we investigate whether the underlying idea can also be transferred to more challenging text-to-text tasks that require the generation of text sequences given an input text, such as abstractive summarization. We introduce GENPET, a novel method based on PET (Schick and Schütze, 2021a), that enables finetuning of generative language models using both instructions and labeled examples. We show that GENPET is a highly data-efficient method that enables us to finetune a pretrained PEGASUS model (Zhang et al., 2020) with as little as 10 or 100 training examples. We evaluate our approach on a diverse set of six English headline generation and text summarization tasks both in zero-shot and few-shot settings and show that PEGASUs trained with GENPET clearly outperforms regular finetuning.

In summary, our contributions are as follows:

- We introduce GENPET, a finetuning procedure for generative language models that achieves great data efficiency by using both textual instructions and training examples.

- We show that training Pegasus with GENPET outperforms standard finetuning across a broad set of tasks and training set sizes.

- We analyze the factors contributing to GENPET's strong performance and quantify the impact of all its components.

\section{Related Work}

Masked language modeling was proposed as a pretraining objective by Devlin et al. (2019). Several variants of this objective that involve generating sequences of text have been proposed, including T5 (Raffel et al., 2020), BART (Lewis et al., 2020) and Pegasus (Zhang et al., 2020), of which we make use in this work.

The idea to rephrase tasks as cloze questions is commonly used to probe the knowledge contained within masked language models (e.g., Petroni et al., 2019; Wang et al., 2019; Talmor et al., 2020; Schick and Schütze, 2020; Ettinger, 2020; Kassner and Schütze, 2020; Sakaguchi et al., 2020). Schick and Schütze (2021a) propose PET, which combines this idea with gradient-based learning for efficient few-shot text classification. Jiang et al. (2020) and Schick et al. (2020) consider the problem of finding the best way to rephrase a given task as a cloze question. Schick and Schütze (2021b)'s version of PET can generate multiple tokens, but still requires a text classification objective and does not scale to long output sequences. Radford et al. (2019) consider task descriptions for text generation tasks, but do so only in a zero-shot setting. In a similar spirit, Brown et al. (2020) investigate the ability of pretrained language models to leverage task descriptions and examples without any gradientbased optimization.

Other approaches to few-shot learning in NLP commonly require large sets of examples from related tasks (Gu et al., 2018; Dou et al., 2019; Qian and Yu, 2019; Ye et al., 2020), parallel data for consistency training (Xie et al., 2020; Chen et al., 2020), or highly specialized methods tailored towards a specific task (Laban et al., 2020). In contrast, GENPET requires no additional labeled data and provides an intuitive interface to leveraging task-specific human knowledge.

Our work is also related to prefix-constrained decoding in interactive machine translation for making suggestions on how to complete a partial translation (Knowles and Koehn, 2016; Wuebker et al., 2016). Keskar et al. (2019) and He et al. (2020) similarly use prompts and keywords for controllable text generation, but require specific pretraining procedures and do so only in high-resource settings.

\section{Pegasus Pretraining}

We briefly summarize the pretraining procedure of Pegasus (Zhang et al., 2020), the model to which we apply GenPet. Pegasus is a standard Transformer encoder-decoder architecture (Vaswani et al., 2017) that is pretrained using gapsentence generation, an objective tailored to text summarization tasks. This pretraining objective requires a set of documents consisting of multi- 


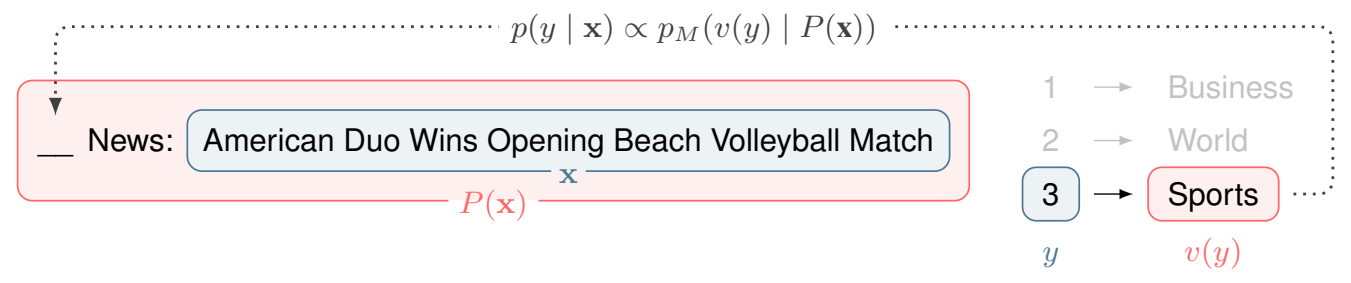

Figure 2: Application of a pattern-verbalizer pair $(P, v)$ in PET: The input $\mathbf{x}$ is converted into a cloze question $P(\mathbf{x})$. The probability $p(y \mid \mathbf{x})$ of each label $y$ is derived from the probability that a pretrained model $M$ assigns to its verbalization $v(y)$ at the masked position. Figure adapted from Schick et al. (2020).

ple sentences. The key idea is to preprocess each document by (i) picking a subset of $m$ informative sentences, ${ }^{2}$ (ii) replacing each of these sentences by a mask token, and (iii) concatenating all removed sentences into a pseudo-summary. The Transformer model is then trained to generate this pseudo-summary given the partially masked document. Similar to prior work (e.g., Raffel et al., 2020; Lewis et al., 2020), this is done by having the encoder process the entire masked document and the decoder generate the output autoregressively.

Zhang et al. (2020) train two variants of PEGASUS: PEgASUS-base, a 12-layer model with approximately 223M parameters, and PEGASUSlarge, a 16-layer model with 568M parameters. As only the latter version is publicly available in a variant that is not finetuned on any downstream task, all our experiments are based on PEGASUS-large.

\section{Pattern-Exploiting Training}

Pattern-Exploiting Training (PET, Schick and Schütze (2021a)) is a finetuning method for text classification tasks. That is, PET can be applied to problems where a text sequence $\mathrm{x} \in \mathcal{X}$ must be mapped to a label $y$ from a finite set $\mathcal{Y}$. As shown in Figure 2, PET enables data-efficient text classification by converting inputs into cloze questions; this drastically reduces the number of examples required (Schick and Schütze, 2021a,b).

Let $M$ be a masked language model, $V$ its vocabulary of tokens and $\_\in V$ the mask token; we denote the set of all token sequences as $V^{*}$. Given an input sequence $\mathbf{z} \in V^{*}$ that contains exactly one mask token, let $p_{M}(t \mid \mathbf{z})$ denote the probability assigned to $t \in V$ by $M$ at the masked position in z. As illustrated in Figure 2, PET requires:

- a pattern $P: \mathcal{X} \rightarrow V^{*}$ that maps each input

\footnotetext{
${ }^{2}$ The most informative sentences are selected where informativeness is measured as the Rouge $1 \mathrm{~F} 1$ score (Lin, 2004) between the sentence and the remaining document.
}

$\mathrm{x}$ to a cloze question containing exactly one mask token;

- a verbalizer $v: \mathcal{Y} \rightarrow V$ that maps each label $y$ to a single token representing its meaning in the pattern.

The probability of $y$ given $\mathbf{x}$ is then derived from the probability that $M$ assigns to $v(y)$ at the masked position in $P(\mathbf{x})$ :

$$
p(y \mid \mathbf{x})=\frac{p_{M}(v(y) \mid P(\mathbf{x}))}{\sum_{y^{\prime} \in \mathcal{Y}} p_{M}\left(v\left(y^{\prime}\right) \mid P(\mathbf{x})\right)}
$$

For finetuning, the cross-entropy between $p(y \mid \mathbf{x})$ and the true label of $\mathbf{x}$ is used as training objective.

\section{Generation with Instructions}

We now introduce GENPET, our method for finetuning language models with instructions for text generation. Similar to PET, we provide instructions by means of patterns $P: \mathcal{X} \rightarrow V^{*}$ that we use to modify the original input. However, we do not require a verbalizer as our output space already consists of natural language sentences, i.e., $\mathcal{Y} \subseteq V^{*}$. In designing GENPET, we tackle three key challenges for few-shot text generation with instructions:

1. How should we provide an instruction to an encoder-decoder model so that the model can make the best possible use of it? (\$5.1)

2. How can we ensure that the model understands the instructions provided sufficiently well, and how do we deal with the fact that even minor modifications to the patterns can have a big impact on performance (Jiang et al., 2020; Schick and Schütze, 2021a; Elazar et al., 2021)? (\$5.2)

3. How do we prevent overfitting, a major issue in few-shot settings? (\$5.3) 
Notation Let $P$ be a pattern, $\mathbf{x} \in \mathcal{X}$ and $\mathbf{y} \in \mathcal{Y}$ input and output text sequences, and $\mathbf{z}=P(\mathbf{x})$ the result of applying $P$ to $\mathrm{x}$, i.e., a text sequence containing a single mask token. Furthermore, let $\mathbf{y}=y_{1} \ldots y_{n}, \mathbf{z}=z_{1} \ldots z_{m}$ and let the mask token in $\mathbf{z}$ be at some position $h \leq m$. We denote the subsequence $y_{i} \ldots y_{j}$ by $\mathbf{y}_{i: j}$.

We consider an encoder-decoder model $M$ pretrained by masked language modeling. That is, the model must be able to compute a probability $p_{M}(\mathbf{y} \mid \mathbf{z})$ that measures to what extent $\mathbf{y}$ is a plausible substitute for the mask in $\mathbf{z}$. We further require that this is done by decomposing the joint probability of $\mathbf{y}$ as follows: ${ }^{3}$

$$
p_{M}(\mathbf{y} \mid \mathbf{z})=\prod_{i=1}^{n} p_{M}\left(y_{i} \mid \mathbf{z} ; \mathbf{y}_{1: i-1}\right)
$$

where $p_{M}\left(y_{i} \mid \mathbf{z} ; \mathbf{y}_{1: i-1}\right)$ is obtained by processing $\mathbf{z}$ using the encoder and $\mathbf{y}_{1: i-1}$ using the decoder. If we happen to already know some prefix $\mathbf{y}_{1: k-1}$ of $\mathbf{y}$, we denote with

$$
p_{M}\left(\mathbf{y}_{k: n} \mid \mathbf{z} ; \mathbf{y}_{1: k-1}\right)=\prod_{i=k}^{n} p_{M}\left(y_{i} \mid \mathbf{z} ; \mathbf{y}_{1: i-1}\right)
$$

the probability that $M$ assigns to the remaining sequence $\mathbf{y}_{k: n}$ if the prefix $\mathbf{y}_{1: k-1}$ was already processed with the decoder.

\subsection{Using a Single Instruction}

As $M$ is an encoder-decoder language model, we have several options for how to apply a pattern $P$, i.e., how to ingest an instruction when computing the probability of $\mathbf{y}$ given $\mathbf{x}$ : We may process the entire sequence $P(\mathbf{x})=\mathbf{z}$ with the encoder, but we may also choose some index $j<h$ and process $\mathbf{z}_{1: j-1} \mathbf{z}_{h: n}$ using the encoder and $\mathbf{z}_{j: h-1}$ using the decoder. For example, if $\mathbf{z}=$ Summary: _ Text: $\mathbf{x}$, we can process the prefix "Summary:" using the encoder or the decoder; that is, we may compute either of the following (cf. Figure 3):

$$
\begin{aligned}
& p_{1}=p_{M}(\mathbf{y} \mid \text { Summary: _ Text: } \mathbf{x}) \\
& p_{2}=p_{M}\left(\mathbf{y} \mid \_ \text {Text: } \mathbf{x} ; \text { Summary: }\right)
\end{aligned}
$$

In preliminary experiments, we found tokens that belong to the partially generated output sequence (i.e., tokens that are processed using the decoder)

\footnotetext{
${ }^{3}$ There are several recent architectures that meet this requirement, including BART (Lewis et al., 2020), T5 (Raffel et al., 2020) and PEGASUs (Zhang et al., 2020).
}
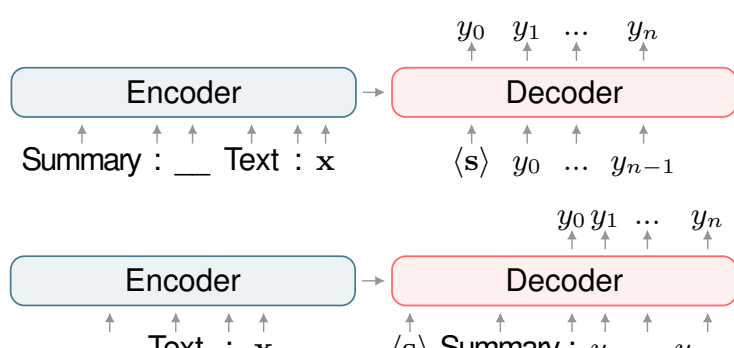

$\langle\mathbf{s}\rangle y_{0} \quad \ldots \quad y_{n-1}$

- Text $: \mathbf{x}$

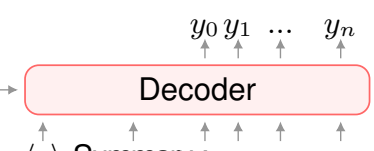

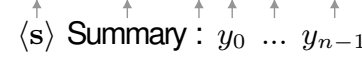

Figure 3: Generation process of an output $\mathbf{y}=y_{0} \ldots y_{n}$ for input $\mathrm{x}$ when the instruction is entirely processed using the encoder (top) and when parts of it are processed using the decoder (bottom). We use $\langle\mathbf{s}\rangle$ to denote the model's start-of-sequence token. The seemingly subtle difference between the two setups can lead to quite different generations: Instructions processed by the decoder have a stronger impact on the model's predictions than those processed by the encoder.

to have a much stronger impact on the model's predictions than regular input tokens (i.e., those processed by the encoder). This applies all the more to PEGASUS, which is pretrained to always generate full sentences: If the pattern used consists of a partial sentence (e.g., a short prompt) which is to be completed by the model, PEGASUs tends to instead simply start a new sentence that does not relate to the given prefix if the latter is processed with the encoder.

Based on this observation, we supplement each pattern $P$ with a decoder prefix $\mathbf{d} \in V^{*}$ that is given to the model as part of the generated sequence rather than the observed input. Accordingly, we define the probability of $\mathbf{y}$ given $\mathbf{x}$ as

$$
p_{(P, \mathbf{d})}(\mathbf{y} \mid \mathbf{x})=p_{M}(\mathbf{y} \mid P(\mathbf{x}) ; \mathbf{d})
$$

In Eqs. 4 and 5, probability $p_{1}$ corresponds to using pattern $P_{1}(\mathbf{x})=$ Summary: _ Text: $\mathbf{x}$ with an empty decoder prefix $\mathbf{d}_{1}$, whereas $p_{2}$ corresponds to using the pattern $P_{2}(\mathbf{x})=\ldots$ Text: $\mathbf{x}$ with a decoder prefix $\mathbf{d}_{2}=$ Summary: . Both variants are illustrated in Figure 3.

We finetune $M$ on a set of training examples $(\mathbf{x}, \mathbf{y})$ simply by minimizing the cross-entropy between $p_{(P, \mathbf{d})}(\mathbf{y} \mid \mathbf{x})$ and $\mathbf{y}$ using teacher forcing.

\subsection{Combining Instructions}

As shown in previous work (Jiang et al., 2020; Schick and Schütze, 2021a), using different instructions or formulating the same input in different ways can have a strong impact on the model's performance. Unfortunately, in the absence of a large 
development set, instructions that work well are often hard to distinguish from those that perform poorly. We alleviate this issue by enabling the simultaneous usage of multiple instructions (represented by multiple pairs of patterns and decoder prefixes) and combining them using a mechanism similar to knowledge distillation (Hinton et al., 2015). This mechanism mitigates the negative influence of instructions that are hard to understand for the model. This means that users can simply provide all (variants of) instructions that they can think of. Further, it is much faster and more memory efficient than having to constantly use multiple instructions (and thus, multiple models) during inference. PET (Schick and Schütze, 2021a) also uses a multi-pattern approach - which is based on averaging the predictions obtained with different patterns -, but it is not applicable in text generation settings as we cannot compute the average of multiple generated sequences in a meaningful way.

Given pairs of patterns and corresponding decoder prefixes $\left(P_{1}, \mathbf{d}_{1}\right), \ldots,\left(P_{k}, \mathbf{d}_{k}\right)$ and a set of models $M_{1}, \ldots, M_{k}$, where each $M_{i}$ was finetuned using $\left(P_{i}, \mathbf{d}_{i}\right)$, we aim to obtain a single model $\tilde{M}$ that contains the combined knowledge of all models. To do so, we require a small set of unlabeled examples $\mathcal{U}$. For each $\mathrm{x} \in \mathcal{U}$, we first generate one output sequence $\mathbf{y}^{\left(P_{i}, \mathbf{d}_{i}\right)}$ per $\left(P_{i}, \mathbf{d}_{i}\right)$ using greedy decoding as in Zhang et al. (2020), resulting in a set of candidate outputs $\mathcal{C}_{\mathbf{x}}=\left\{\mathbf{y}^{\left(P_{i}, \mathbf{d}_{i}\right)} \mid 1 \leq i \leq k\right\}$. To assign a score to each candidate $\mathbf{y} \in \mathcal{C}_{\mathbf{x}}$, we first compute the log-likelihood of $\mathbf{y}$ for each $\left(P_{i}, \mathbf{d}_{i}\right)$ as

$$
s_{i}(\mathbf{y} \mid \mathbf{x})=\log p_{\left(P_{i}, \mathbf{d}_{i}\right)}(\mathbf{y} \mid \mathbf{x})
$$

The total score of $\mathbf{y}$ is then simply the exponentiated average over the patterns:

$$
s(\mathbf{y} \mid \mathbf{x})=\exp \frac{1}{k} \sum_{i=1}^{k} s_{i}(\mathbf{y} \mid \mathbf{x})
$$

The model $\tilde{M}$ is trained on pairs $(\mathbf{x}, \mathbf{y})$ where $\mathrm{x} \in \mathcal{U}$ and $\mathbf{y}$ is drawn from $\mathcal{C}_{\mathbf{x}}$ with probability proportional to $s(\mathbf{y} \mid \mathbf{x})$.

While we could train this final model to simply maximize $p_{\tilde{M}}(\mathbf{y} \mid \mathbf{x})$, we note that this creates a large discrepancy between pretraining and finetuning: During pretraining, masked language models only process sequences that contain at least one mask token. In the spirit of our intention to make pretraining and finetuning as similar as possible $(\S 1)$, we therefore train $\widetilde{M}$ using a trivial pattern
$P(\mathbf{x})=\ldots \mathrm{x}$ that just prepends a single mask token to the input and use an empty decoder prefix; that is, we maximize $p_{\tilde{M}}(\mathbf{y} \mid \ldots \mathbf{x} ;)$ instead of $p_{\tilde{M}}(\mathbf{y} \mid \mathbf{x})$. In addition to reducing the pretrainingfinetuning discrepancy, putting the mask token $b e$ fore the input biases the model towards generating text that is likely to precede the input. This is desirable because news articles - which abound in big language models' pretraining data - often have a headline and a short summary before the article rather than after it.

\subsection{Preventing Overfitting}

In preliminary experiments, we found pretrained encoder-decoder models to strongly overfit the training data when trained on just a handful of examples: When generating new texts, they often simply reproduce phrases from training examples, even if they are not in any way related to the current input. To alleviate this issue, we introduce two modifications to our training procedure; we refer to them as unsupervised scoring and joint training.

Unsupervised Scoring For unsupervised scoring, we compute $s(\mathbf{y} \mid \mathbf{x})$ as in Eq. 8, but we use an untrained model (i.e., one that has not been finetuned on task-specific examples) to compute $p_{\left(P_{i}, \mathbf{d}_{i}\right)}(\mathbf{y} \mid \mathbf{x})$ in Eq. 7 for all $i \in\{1, \ldots, k\}$.

The intuition behind this is as follows: If for a given input, a trained model simply reproduces phrases from its training set, the resulting pair of input and output texts should look strange to an untrained model, which has not seen the example from which the output is (partially) copied. Thus, sampling outputs from the candidate set $\mathcal{C}_{\mathbf{x}}$ based on the probability assigned to each example by an untrained model helps prevent overfitting: It results in the final model being primarily trained on examples that also look natural to a model that has not seen the training data.

We further use this idea to discard generated texts of really poor quality altogether. To this end, we sort the set $\mathcal{C}=\bigcup_{\mathbf{x} \in \mathcal{U}} \mathcal{C}_{\mathbf{x}}$ of all outputs for all candidate sets based on their likelihood according to the untrained model in ascending order. Let the rank $r_{\mathbf{y}}$ of each output $\mathbf{y} \in \mathcal{C}$ be its position in this sorted list, divided by the list's size. We then remove all outputs with $r_{\mathbf{y}}<\tau$ from the candidate sets $\mathcal{C}_{\mathbf{x}}$, where the threshold $\tau$ is a hyperparameter.

Joint Training In §5.2, we assume the existence of an ensemble $\left\{M_{1}, \ldots, M_{k}\right\}$ where each model 
was trained using a different instruction. However, instead of training an individual model $M_{i}$ for each pair $\left(P_{i}, \mathbf{d}_{i}\right)$, we can also train a single model jointly on all instructions. To do so, we simply replicate each training instance $k$ times and process the $i$ th copy with $\left(P_{i}, \mathbf{d}_{i}\right)$. Our motivation is that forcing a single model to work well for all instructions can act as a regularizer to prevent overfitting. This approach comes with the additional benefits of both being faster to train and generating less overhead. Note that we still require instruction combination ( $\$ 5.2)$ because even given a single model understanding all instructions, it would be unclear which instruction to choose during test time, and querying the model with all instructions would be inefficient.

\section{Experiments}

Tasks We evaluate Pegasus with and without GENPET on a subset of the tasks in Zhang et al. (2020). As our computing resources are limited, we only choose those tasks for which the maximum output length in Zhang et al. (2020) is at most 128 tokens. We include the following tasks:

- AESLC (Zhang and Tetreault, 2019): Given an email body, predict the title of the email.

- Gigaword (Rush et al., 2015): Given the first sentence of a news article, generate its headline.

- XSum (Narayan et al., 2018): Summarize articles spanning a wide range of different topics.

- Reddit TIFU (Kim et al., 2019): Generate summaries for posts from the TIFU community in Reddit.

- NEWSROOM (Grusky et al., 2018): Generate summaries for articles from various major publications.

- CNN/DailyMail (Hermann et al., 2015): For articles from CNN and the Daily Mail, generate a list of highlights.

For each task, we use the entire test set for evaluation. ${ }^{4}$ We create two types of training sets containing either 10 or 100 training examples; in addition, we provide 1,000 unlabeled examples per

\footnotetext{
${ }^{4}$ The only exception to this is NEWSROOM, which contains more than 100,000 examples: We only consider a subset of 10,000 examples to ensure a resource-friendly evaluation.
}

\begin{tabular}{lll}
\hline Task & Decoder Prefixes & \\
\hline AESLC & $d_{1}=$ E-Mail Subject: & $d_{2}=$ E-Mail Topic: \\
Gigaword & $d_{1}=$ Headline: & $d_{2}=$ Article Headline: \\
CNN/DM & $d_{1}=$ Highlights: & $d_{2}=$ Article Highlights: \\
Others & $d_{1}=$ Short Summary: & $d_{2}=$ Brief Summary: \\
\hline
\end{tabular}

Table 1: Decoder prefixes we use for AESLC, Gigaword, CNN/DailyMail (CNN/DM) and all other summarization tasks (Others)

task. Both unlabeled and training examples are obtained through uniform sampling from each task's original training set. ${ }^{5}$

As previous work (Schick and Schütze, 2021b) has shown that the choice of training examples has a large impact on model performance, we create three distinct training sets per size (10 and 100) and task using different random seeds, resulting in a total of six training sets per task. Scores reported in this section are always average scores across all three equal-sized sets of training examples, except for zero-shot settings where no training data is available at all.

Instructions We use the same set of patterns across all tasks, but we combine them with different decoder prefixes. The patterns we use are:

$$
P_{1}(\mathbf{x})=\ldots \mathbf{x} \quad P_{2}(\mathbf{x})={ }_{-} \text {Text: } \mathbf{x}
$$

All decoder prefixes are shown in Table 1. We combine each pattern with each decoder prefix, resulting in four pairs per task: $\left(P_{1}, d_{1}\right),\left(P_{1}, d_{2}\right)$, $\left(P_{2}, d_{1}\right),\left(P_{2}, d_{2}\right)$.

Setup For all our experiments with GENPET, we use PEgAsus-large (Zhang et al., 2020) as underlying language model and perform greedy decoding; our implementation is based on the Transformers library (Wolf et al., 2020) and PyTorch (Paszke et al., 2017). Unless stated differently, all experiments are performed using the same setup as Schick and Schütze (2021a) and a single GPU with 11GB RAM (NVIDIA GeForce GTX 1080 Ti).

For optimizing hyperparameters, much previous few-shot work uses development sets that are larger than the training sets by multiple orders of magnitude (e.g., Xie et al., 2020; Zhang et al., 2020; Chen et al., 2020); however, assuming the existence of such large development sets is inconsistent with real-world few-shot settings. In contrast, Schick

\footnotetext{
${ }^{5}$ We do not reuse the datasets of Zhang et al. (2020) as they did not use a fixed seed and thus their training data is not recoverable.
} 


\begin{tabular}{|c|c|c|c|c|c|c|c|c|}
\hline$t$ & Model & AESLC & Gigaword & XSum & Reddit TIFU & NEWSROOM & CNN/DailyMail & Avg \\
\hline \multirow{3}{*}{0} & Pegasus & $8.20 / 2.74 / 7.35$ & $23.91 / 7.66 / 20.64$ & $18.61 / 2.54 / 12.06$ & $17.19 / 3.29 / 12.00$ & $23.24 / 11.20 / 18.34$ & $\mathbf{3 5 . 2 0 / 1 4 . 0 7 / 2 2 . 8 4}$ & $21.06 / 6.91 / 15.54$ \\
\hline & PEgASUS-M & $12.39 / 4.74 / 11.42$ & $19.63 / 5.51 / 16.97$ & $32.43 / 13.10 / 24.58$ & $14.80 / 2.89 / 10.74$ & $25.01 / 13.57 / 20.90$ & $33.36 / 12.97 / 22.63$ & $22.94 / 8.80 / 17.87$ \\
\hline & GENPET & $19.81 / 8.81 / 18.53$ & 28.01/10.48/24.92 & $29.24 / 10.56 / 22.73$ & $15.41 / 2.83 / 11.63$ & $26.35 / 15.79 / 23.22$ & $33.08 / 12.82 / 23.27$ & $25.32 / 10.21 / 20.71$ \\
\hline \multirow{3}{*}{10} & PEgasus & $9.37 / 3.77 / 8.97$ & $25.18 / 9.24 / 22.80$ & $30.41 / 9.57 / 23.26$ & $18.48 / 3.97 / 14.08$ & $25.59 / 12.28 / 21.18$ & $37.54 / 15.84 / 25.18$ & $24.43 / 9.11 / 19.24$ \\
\hline & PEgASUS-M & $16.53 / 7.47 / 16.15$ & $27.33 / 10.60 / 24.98$ & $33.96 / 11.90 / 26.29$ & $19.78 / 4.50 / 15.16$ & $29.91 / 16.73 / 25.70$ & $37.88 / 16.19 / 25.82$ & $27.56 / 11.23 / 22.35$ \\
\hline & GENPET & $27.19 / 14.08 / 26.73$ & $30.93 / 13.02 / 28.49$ & $35.88 / 13.22 / 28.24$ & $22.43 / 5.55 / 17.27$ & $34.48 / 22.00 / 30.60$ & $38.91 / 16.97 / 26.65$ & 31.63/14.14/26.33 \\
\hline \multirow{3}{*}{100} & PEgasus & $23.22 / 10.24 / 22.43$ & $30.80 / 12.27 / 27.92$ & $40.23 / 16.68 / 31.90$ & $24.24 / 6.28 / 18.72$ & $33.13 / 20.24 / 28.80$ & $39.64 / 16.94 / 26.79$ & $31.87 / 13.77 / 26.10$ \\
\hline & Pegasus-M & $25.87 / 12.34 / 24.99$ & $31.38 / 12.65 / 28.33$ & $40.73 / 17.10 / 32.43$ & $24.74 / 6.40 / 19.10$ & $34.79 / 21.60 / 30.37$ & 40.08/17.14/27.06 & $32.93 / 14.54 / 27.05$ \\
\hline & GENPET & $29.97 / 15.32 / 29.26$ & $32.75 / 13.98 / 29.94$ & 41.71/17.99/33.46 & $26.06 / 7.34 / 20.34$ & 36.20/23.51/32.02 & $40.02 / \mathbf{1 7 . 7 7 / 2 7 . 7 9}$ & $34.45 / 15.98 / 28.80$ \\
\hline
\end{tabular}

Table 2: R1/R2/RL scores for six tasks and three training set sizes $t$; for 10 and 100 examples, all results are averaged across three different (seed-dependent) training sets. The last column shows average performance across all tasks.

and Schütze (2021a) assume no development data at all and determine hyperparameters based only on previous work and practical considerations. We choose a middle course and create a small development set of 100 examples for only one of the six tasks, XSum. We use this development set in combination with a single training set of 10 examples to determine hyperparameters for all tasks and training sets. However, we do so only for hyperparameters for which no consistent value can be derived from previous work.

Following Zhang et al. (2020), we use a maximum input length of 512 tokens, the Adafactor optimizer (Shazeer and Stern, 2018) with square root learning rate decay, a dropout rate of 0.1 and label smoothing setting $\varepsilon=0.1$ (Szegedy et al., 2016); we also adopt Zhang et al. (2020)'s maximum output lengths for each task. As recommended by Schick and Schütze (2021a), we train all models for 250 steps using a batch size of 8 . We also tried training for 500 and 1,000 steps on our development set but found no major differences in performance. For the learning rate, we tried values of $\alpha \cdot 10^{-5}$ with $\alpha \in\{1,10,50\}$ as Schick and Schütze (2021a) use $\alpha=1$ and Zhang et al. (2020) use $\alpha=50$; we found $\alpha=10$ to perform best for all models. For unsupervised scoring ( 55.3$)$, we use a threshold of $\tau=0.2$, i.e., we discard the $20 \%$ of examples that are least likely according to an untrained model. We chose this value by looking at texts generated by PEGASUS trained on 10 examples from the XSum development set, where we found the bottom $20 \%$ to contain texts of poor quality, including random telephone numbers and repetitions of the same word. For evaluation, we follow Zhang et al. (2020) and report Rouge1, Rouge 2 and RougeL (R1/R2/RL) F1 scores (Lin, 2004) after stemming using the Porter algorithm (Porter, 1997).
Results On all six tasks, we compare the following three approaches for finetuning a pretrained Pegasus model:

- Pegasus: The regular finetuning procedure described in (Zhang et al., 2020).

- Pegasus-M: Finetuning with a single trivial pattern that inserts a mask token before the first word.

- GENPET: Finetuning with GENPET using patterns $P_{1}$ and $P_{2}$ and the decoder prefixes in Table 1 as described above; we apply all modifications described in $\$ 5.3$.

We do not compare to other few-shot approaches as they either make quite different assumptions for example, GENPET requires manually designed patterns and some amount of unlabeled examples, whereas meta learning approaches (e.g., Gu et al., 2018; Dou et al., 2019; Qian and Yu, 2019) require large annotated datasets for related tasks -, or they cannot be transferred to a generative setting in a straightforward fashion, as is the case for consistency-based methods such as those of Xie et al. (2020) and Chen et al. (2020). However, we note that PEGASUS is a strong baseline in terms of data efficiency, almost matching the performance of prior state-of-the-art systems trained on the full datasets with as little as 100 examples for many tasks (Zhang et al., 2020).

Table 2 shows results for zero-shot learning and for few-shot learning with 10 and 100 training examples. In the few-shot settings, GENPET consistently outperforms PEGASUS across all tasks, resulting in an average improvement in $\mathrm{R} 1$ over PEgASUS of 7.20 (31.63 vs 24.43) and 2.58 (34.45 vs 31.87). PEgASUS-M performs better than regular finetuning, indicating that even just adding 
a single mask token at the very beginning, without any instructions, already effectively improves performance. (Recall that the effect of the initial mask is to make finetuning more similar to pretraining and to bias the models towards generating text that is likely to appear before the input; see §5.2). However, it still performs clearly worse than GENPET, demonstrating that PEGASUS is indeed able to make use of the instructions provided. In the zero-shot setting, GENPET also outperforms all baselines on average, but falls short on individual tasks.

Quantitative Analysis To analyze the factors contributing to GENPET's performance, Table 3 compares the performance of the best ("best only") and the worst ("worst only") performing pairs of pattern and decoder prefix to that of GENPET in a setting with 10 training examples. We see some difference in performance between using only the best and worst pairs, but this difference is not as pronounced as in previous work (Schick and Schütze, 2021b,a) - possibly because our instructions are more similar to each other than patterns in prior work. Notably, our strategy for combining instructions clearly performs better than using just the best instruction across all tasks and measures (compare GENPET with "best only"). Table 3 also shows results for using the best pattern without a decoder prefix ("no dec. prefix") and instead processing the entire input using the encoder. That is, given $(P, \mathbf{d})$ with $P(\mathbf{x})=z_{1} \ldots z_{n}$ and $z_{h}=$ we compute $p_{M}\left(\mathbf{y} \mid z_{1} \ldots z_{h-1} \mathbf{d} z_{h} \ldots z_{n}\right)$ rather than $p_{M}\left(\mathbf{y} \mid z_{1} \ldots z_{n} ; \mathbf{d}\right)$ similar to the example shown in Figure 3 (top). While this variant still performs better than PEGASUS-M on two out of three datasets, results clearly show that PEGASUS makes less use of task descriptions if they are processed using the encoder.

The bottom two rows of Table 3 show performance when we replace unsupervised scoring (§5.3) with regular scoring using the supervised models ("sup. scoring") and if we additionally do not perform joint training ("no joint train."). As can be seen, not using joint training hurts performance for all three tasks and supervised scoring hurts performance for two out of three tasks.

Qualitative Analysis Table 4 shows zero-shot abilities of three methods for one selected input from Gigaword that illustrates some typical behaviors: Regular Pegasus just creates a verbatim

\begin{tabular}{|c|c|c|c|}
\hline Model & AESLC & XSum & NEWSROOM \\
\hline Pegasu & $9.37 / 3.77$ & $30.41 / 9$ & 25.5 \\
\hline PEgasus-M & $16.53 / 7.47 / 16.15$ & $33.96 / 11.90 / 26.29$ & $29.91 / 16.73 / 25.70$ \\
\hline GENPET & $27.19 / 14.08 / 26.73$ & $35.88 / 13.22 / 28.24$ & $34.48 / 2$ \\
\hline$L$ worst only & $24.08 / 12.22 / 23.58$ & $33.85 / 11.95 / 26.60$ & $32.55 / 19.73 / 28.59$ \\
\hline$L$ best only & $24.80 / 12.48 / 24.19$ & $34.15 / 12.05 / 26.78$ & $33.94 / 21.34 / 30.03$ \\
\hline$L_{\text {no dec. prefix }}$ & $15.49 / 7.24 / 15.09$ & $34.12 / 11.95 / 26.41$ & $32.56 / 20.15 / 28.64$ \\
\hline $\mathrm{L}$ sup. scoring & $25.33 / 13.41 / 24.87$ & $35.68 / 13.19 / 28.06$ & $34.37 / 22.04 / 30.53$ \\
\hline$L$ no joint train. & $24.37 / 12.67 / 24.00$ & $35.41 / 13.15 / 27.95$ & $34.04 / 21.95 / 30.35$ \\
\hline
\end{tabular}

Table 3: R1/R2/RL scores for several baselines and variants of GENPET given 10 training examples

Input: the dollar slipped against the euro on friday after the u.s. federal reserve cut its discount rate to banks by a half percentage point.

PG federal reserve cut its discount rate to banks by a half percentage point.

PG-M The dollar fell against the euro on monday after the u.s.

GENPET dollar slips against euro after federal reserve cuts discount rate to banks.

Gold dollar slides against euro as fed cuts discount rate

Table 4: Zero-shot summaries for the news item given as "Input". PEGASUS (PG) simply creates a verbatim copy of the second part of the input. PEGASUS-M (PGM) hallucinates ("Monday" vs. "Friday"). GENPET's summary is close in quality to gold.

copy of the input's second half - this is true not only for this particular example, but can be seen frequently for all datasets. We assume this is due to the fact that Zhang et al. (2020) introduce some modifications to their training procedure that encourage the model to copy text. PEgASUS-M is able to produce an output that is not just a wordfor-word copy of the input, but hallucinates information that is not backed by the input text ("monday"). We found that hallucination is a frequent problem for PEGASUS-M. This is hardly surprising given that the model has no way of knowing that it is expected to generate a factual headline summarizing the input. In contrast, GENPET generates a fluent and factual headline that covers all relevant aspects.

\section{Conclusion}

We investigated the ability of pretrained language models to make use of simple instructions with the aim of enabling more data-efficient text generation. We identified three major challenges: enabling language models to make good use of the instructions provided, ensuring that the instructions are useful and preventing overfitting. We tackle these in our proposed approach, GENPET, by (i) introducing 
the concept of decoder prefixes, (ii) combining instructions through knowledge distillation where target sequences are generated with probabilistically sampled instructions and (iii) making use of unsupervised scoring and joint training. A pretrained PEGASUS model finetuned with GENPET clearly outperforms regular finetuning in few-shot settings.

Acknowledgments This work was funded by the European Research Council (ERC \#740516). We thank the anonymous reviewers for their helpful comments.

\section{References}

Tom Brown, Benjamin Mann, Nick Ryder, Melanie Subbiah, Jared D Kaplan, Prafulla Dhariwal, Arvind Neelakantan, Pranav Shyam, Girish Sastry, Amanda Askell, Sandhini Agarwal, Ariel Herbert-Voss, Gretchen Krueger, Tom Henighan, Rewon Child, Aditya Ramesh, Daniel Ziegler, Jeffrey Wu, Clemens Winter, Chris Hesse, Mark Chen, Eric Sigler, Mateusz Litwin, Scott Gray, Benjamin Chess, Jack Clark, Christopher Berner, Sam McCandlish, Alec Radford, Ilya Sutskever, and Dario Amodei. 2020. Language models are few-shot learners. In $A d$ vances in Neural Information Processing Systems, volume 33, pages 1877-1901. Curran Associates, Inc.

Jiaao Chen, Zichao Yang, and Diyi Yang. 2020. MixText: Linguistically-informed interpolation of hidden space for semi-supervised text classification. In Proceedings of the 58th Annual Meeting of the Association for Computational Linguistics, pages 21472157, Online. Association for Computational Linguistics.

Jacob Devlin, Ming-Wei Chang, Kenton Lee, and Kristina Toutanova. 2019. BERT: Pre-training of deep bidirectional transformers for language understanding. In Proceedings of the 2019 Conference of the North American Chapter of the Association for Computational Linguistics: Human Language Technologies, Volume 1 (Long and Short Papers), pages 4171-4186, Minneapolis, Minnesota. Association for Computational Linguistics.

Zi-Yi Dou, Keyi Yu, and Antonios Anastasopoulos. 2019. Investigating meta-learning algorithms for low-resource natural language understanding tasks. In Proceedings of the 2019 Conference on Empirical Methods in Natural Language Processing and the 9th International Joint Conference on Natural Language Processing (EMNLP-IJCNLP), pages 1192 1197, Hong Kong, China. Association for Computational Linguistics.

Yanai Elazar, Nora Kassner, Shauli Ravfogel, Abhilasha Ravichander, Eduard Hovy, Hinrich Schütze, and
Yoav Goldberg. 2021. Measuring and improving consistency in pretrained language models. Computing Research Repository, arXiv:2102.01017.

Allyson Ettinger. 2020. What BERT is not: Lessons from a new suite of psycholinguistic diagnostics for language models. Transactions of the Association for Computational Linguistics, 8:34-48.

Max Grusky, Mor Naaman, and Yoav Artzi. 2018. Newsroom: A dataset of 1.3 million summaries with diverse extractive strategies. Proceedings of the 2018 Conference of the North American Chapter of the Association for Computational Linguistics: Human Language Technologies, Volume 1 (Long Papers).

Jiatao Gu, Yong Wang, Yun Chen, Victor O. K. Li, and Kyunghyun Cho. 2018. Meta-learning for lowresource neural machine translation. In Proceedings of the 2018 Conference on Empirical Methods in Natural Language Processing, pages 3622-3631, Brussels, Belgium. Association for Computational Linguistics.

Junxian He, Wojciech Kryściński, Bryan McCann, Nazneen Rajani, and Caiming Xiong. 2020. CTRLsum: Towards generic controllable text summarization. Computing Research Repository, arXiv:2012.04281.

Karl Moritz Hermann, Tomas Kocisky, Edward Grefenstette, Lasse Espeholt, Will Kay, Mustafa Suleyman, and Phil Blunsom. 2015. Teaching machines to read and comprehend. In Advances in Neural Information Processing Systems, volume 28, pages 1693-1701. Curran Associates, Inc.

Geoffrey Hinton, Oriol Vinyals, and Jeff Dean. 2015. Distilling the knowledge in a neural network. Computing Research Repository, arXiv:1503.02531.

Jeremy Howard and Sebastian Ruder. 2018. Universal language model fine-tuning for text classification. In Proceedings of the 56th Annual Meeting of the Association for Computational Linguistics (Volume 1: Long Papers), pages 328-339, Melbourne, Australia. Association for Computational Linguistics.

Zhengbao Jiang, Frank F. Xu, Jun Araki, and Graham Neubig. 2020. How can we know what language models know? Transactions of the Association for Computational Linguistics, 8:423-438.

Nora Kassner and Hinrich Schütze. 2020. Negated and misprimed probes for pretrained language models: Birds can talk, but cannot fly. In Proceedings of the 58th Annual Meeting of the Association for Computational Linguistics, pages 7811-7818, Online. Association for Computational Linguistics.

Nitish Shirish Keskar, Bryan McCann, Lav R Varshney, Caiming Xiong, and Richard Socher. 2019. CTRL: A conditional transformer language model for controllable generation. Computing Research Repository, arXiv:1909.05858. 
Byeongchang Kim, Hyunwoo Kim, and Gunhee Kim. 2019. Abstractive summarization of Reddit posts with multi-level memory networks. In Proceedings of the 2019 Conference of the North American Chapter of the Association for Computational Linguistics. Human Language Technologies, Volume 1 (Long and Short Papers), pages 2519-2531, Minneapolis, Minnesota. Association for Computational Linguistics.

Rebecca Knowles and Philipp Koehn. 2016. Neural interactive translation prediction. In Proceedings of the Association for Machine Translation in the Americas, pages 107-120.

Philippe Laban, Andrew Hsi, John Canny, and Marti A Hearst. 2020. The summary loop: Learning to write abstractive summaries without examples. In Proceedings of the 58th Annual Meeting of the Association for Computational Linguistics, pages 5135-5150, Online. Association for Computational Linguistics.

Haejun Lee, Drew A. Hudson, Kangwook Lee, and Christopher D. Manning. 2020. SLM: Learning a discourse language representation with sentence unshuffling. In Proceedings of the 2020 Conference on Empirical Methods in Natural Language Processing (EMNLP), pages 1551-1562, Online. Association for Computational Linguistics.

Mike Lewis, Yinhan Liu, Naman Goyal, Marjan Ghazvininejad, Abdelrahman Mohamed, Omer Levy, Veselin Stoyanov, and Luke Zettlemoyer. 2020. BART: Denoising sequence-to-sequence pre-training for natural language generation, translation, and comprehension. In Proceedings of the 58th Annual Meeting of the Association for Computational Linguistics, pages 7871-7880, Online. Association for Computational Linguistics.

Chin-Yew Lin. 2004. ROUGE: A package for automatic evaluation of summaries. In Text Summarization Branches Out, pages 74-81, Barcelona, Spain. Association for Computational Linguistics.

Stephen Mayhew, Gupta Nitish, and Dan Roth. 2020. Robust named entity recognition with truecasing pretraining. Proceedings of the AAAI Conference on Artificial Intelligence, 34(05):8480-8487.

Shashi Narayan, Shay B. Cohen, and Mirella Lapata. 2018. Don't give me the details, just the summary! topic-aware convolutional neural networks for extreme summarization. In Proceedings of the 2018 Conference on Empirical Methods in Natural Language Processing, pages 1797-1807, Brussels, Belgium. Association for Computational Linguistics.

Adam Paszke, Sam Gross, Soumith Chintala, Gregory Chanan, Edward Yang, Zachary DeVito, Zeming Lin, Alban Desmaison, Luca Antiga, and Adam Lerer. 2017. Automatic differentiation in PyTorch. In NIPS Autodiff Workshop.

Matthew Peters, Mark Neumann, Mohit Iyyer, Matt Gardner, Christopher Clark, Kenton Lee, and Luke
Zettlemoyer. 2018. Deep contextualized word representations. In Proceedings of the 2018 Conference of the North American Chapter of the Association for Computational Linguistics: Human Language Technologies, Volume 1 (Long Papers), pages 2227-2237, New Orleans, Louisiana. Association for Computational Linguistics.

Fabio Petroni, Tim Rocktäschel, Sebastian Riedel, Patrick Lewis, Anton Bakhtin, Yuxiang Wu, and Alexander Miller. 2019. Language models as knowledge bases? Proceedings of the 2019 Conference on Empirical Methods in Natural Language Processing and the 9th International Joint Conference on Natural Language Processing (EMNLP-IJCNLP).

Martin F. Porter. 1997. An Algorithm for Suffix Stripping, page 313-316. Morgan Kaufmann Publishers Inc., San Francisco, CA, USA.

Kun Qian and Zhou Yu. 2019. Domain adaptive dialog generation via meta learning. In Proceedings of the 57th Annual Meeting of the Association for Computational Linguistics, pages 2639-2649, Florence, Italy. Association for Computational Linguistics.

Alec Radford, Karthik Narasimhan, Tim Salimans, and Ilya Sutskever. 2018. Improving language understanding by generative pre-training.

Alec Radford, Jeff Wu, Rewon Child, David Luan, Dario Amodei, and Ilya Sutskever. 2019. Language models are unsupervised multitask learners. Technical report.

Colin Raffel, Noam Shazeer, Adam Roberts, Katherine Lee, Sharan Narang, Michael Matena, Yanqi Zhou, Wei Li, and Peter J. Liu. 2020. Exploring the limits of transfer learning with a unified text-to-text transformer. Journal of Machine Learning Research, 21(140):1-67.

Alexander M. Rush, Sumit Chopra, and Jason Weston. 2015. A neural attention model for abstractive sentence summarization. In Proceedings of the 2015 Conference on Empirical Methods in Natural Language Processing, pages 379-389, Lisbon, Portugal. Association for Computational Linguistics.

Keisuke Sakaguchi, Ronan Le Bras, Chandra Bhagavatula, and Yejin Choi. 2020. WinoGrande: An adversarial winograd schema challenge at scale. In Proceedings of the Thirty-Fourth AAAI Conference on Artificial Intelligence.

Timo Schick, Helmut Schmid, and Hinrich Schütze. 2020. Automatically identifying words that can serve as labels for few-shot text classification. In Proceedings of the 28th International Conference on Computational Linguistics, pages 5569-5578, Barcelona, Spain (Online). International Committee on Computational Linguistics.

Timo Schick and Hinrich Schütze. 2020. Rare words: A major problem for contextualized embeddings and how to fix it by attentive mimicking. In Proceedings 
of the Thirty-Fourth AAAI Conference on Artificial Intelligence.

Timo Schick and Hinrich Schütze. 2021a. Exploiting cloze questions for few shot text classification and natural language inference. In Proceedings of the 16th Conference of the European Chapter of the Association for Computational Linguistics, Kyiv, Ukraine (Online). International Committee on Computational Linguistics.

Timo Schick and Hinrich Schütze. 2021b. It's not just size that matters: Small language models are also fewshot learners. In Proceedings of the 2021 Conference of the North American Chapter of the Association for Computational Linguistics: Human Language Technologies, pages 2339-2352, Online. Association for Computational Linguistics.

Noam Shazeer and Mitchell Stern. 2018. Adafactor: Adaptive learning rates with sublinear memory cost. In Proceedings of the 35th International Conference on Machine Learning, volume 80 of Proceedings of Machine Learning Research, pages 4596-4604. PMLR.

C. Szegedy, V. Vanhoucke, S. Ioffe, J. Shlens, and Z. Wojna. 2016. Rethinking the inception architecture for computer vision. In 2016 IEEE Conference on Computer Vision and Pattern Recognition (CVPR), pages 2818-2826.

Alon Talmor, Yanai Elazar, Yoav Goldberg, and Jonathan Berant. 2020. oLMpics-on what language model pre-training captures. Transactions of the Association for Computational Linguistics, 8:743-758.

Ashish Vaswani, Noam Shazeer, Niki Parmar, Jakob Uszkoreit, Llion Jones, Aidan N Gomez, Lukasz Kaiser, and Illia Polosukhin. 2017. Attention is all you need. In Advances in Neural Information Processing Systems 30, pages 5998-6008. Curran Associates, Inc.

Cunxiang Wang, Shuailong Liang, Yue Zhang, Xiaonan Li, and Tian Gao. 2019. Does it make sense? And why? A pilot study for sense making and explanation. In Proceedings of the 57th Annual Meeting of the Association for Computational Linguistics, pages 4020-4026, Florence, Italy. Association for Computational Linguistics.

Thomas Wolf, Lysandre Debut, Victor Sanh, Julien Chaumond, Clement Delangue, Anthony Moi, Pierric Cistac, Tim Rault, Remi Louf, Morgan Funtowicz, Joe Davison, Sam Shleifer, Patrick von Platen, Clara Ma, Yacine Jernite, Julien Plu, Canwen Xu, Teven Le Scao, Sylvain Gugger, Mariama Drame, Quentin Lhoest, and Alexander Rush. 2020. Transformers: State-of-the-art natural language processing. In Proceedings of the 2020 Conference on Empirical Methods in Natural Language Processing: System Demonstrations, pages 38-45, Online. Association for Computational Linguistics.
Joern Wuebker, Spence Green, John DeNero, Saša Hasan, and Minh-Thang Luong. 2016. Models and inference for prefix-constrained machine translation. In Proceedings of the 54th Annual Meeting of the Association for Computational Linguistics (Volume 1: Long Papers), pages 66-75, Berlin, Germany. Association for Computational Linguistics.

Qizhe Xie, Zihang Dai, Eduard Hovy, Thang Luong, and Quoc Le. 2020. Unsupervised data augmentation for consistency training. In Advances in Neural Information Processing Systems, volume 33, pages 6256-6268. Curran Associates, Inc.

Zhiquan Ye, Yuxia Geng, Jiaoyan Chen, Jingmin Chen, Xiaoxiao Xu, SuHang Zheng, Feng Wang, Jun Zhang, and Huajun Chen. 2020. Zero-shot text classification via reinforced self-training. In Proceedings of the 58th Annual Meeting of the Association for Computational Linguistics, pages 3014-3024, Online. Association for Computational Linguistics.

Jingqing Zhang, Yao Zhao, Mohammad Saleh, and Peter Liu. 2020. PEGASUS: Pre-training with extracted gap-sentences for abstractive summarization. In Proceedings of the 37th International Conference on Machine Learning, volume 119 of Proceedings of Machine Learning Research, pages 11328-11339, Virtual. PMLR.

Rui Zhang and Joel Tetreault. 2019. This email could save your life: Introducing the task of email subject line generation. In Proceedings of the 57th Annual Meeting of the Association for Computational Linguistics, pages 446-456, Florence, Italy. Association for Computational Linguistics. 


\section{A Analysis}

Sequence Length We look at the performance of GENPET as a function of the maximum output length $\ell$. One might be concerned that the influence of the decoder prefix on generated tokens may decrease with distance. This would mean that diminishing gains are to be expected from GENPET for tasks that require longer text sequences to be generated. To investigate whether this is a problem for GENPET, Table 5 shows the performance of PEGASUS and GENPET for all tasks with an original maximum output length of 128 tokens, using maximum output lengths of $\ell=32$ and 128 .

For both values of $\ell$, we compute the gains $g_{\ell}$ from using GENPET as the difference in performance between GENPET and PEGASUS. On average, increasing $\ell$ to 128 tokens reduces the gains from GENPET over regular finetuning by just $g_{32}-g_{128}=0.10$ points R1. This shows that instructions provided using GENPET have a strong impact on generated tokens even if there are dozens of other tokens in between. Thus, GENPET works not only for short sequences, but is also beneficial for generating long text sequences.

Unsupervised Scoring We motivated the use of unsupervised scoring in Section 5.2 by the observation that PEGASUS tends to overfit the training data. This can for example be seen when training PEgAsus with individual instructions on the 10 examples from the XSum dataset used to optimize hyperparameters. One of these examples has the gold-standard summary "Hugo Chavez [...] is one of the most visible, vocal and controversial leaders in Latin America"; as shown in Table 6, this induces PEGASUS to generate the phrase "the most visible, vocal and controversial" for many other inputs, even in cases where this phrase does not make any sense given the input text. Out of the summaries generated for 1,000 unlabeled examples, we found 92 to contain this particular phrase word-for-word.

Table 6 also shows the rank of each output as defined in Section 5.3 (i.e., its relative position in a list of all generated outputs that is sorted by likelihood in ascending order) both when likelihood is assigned using the trained models $\left(r_{\text {sup }}\right)$ and when it is assigned using a fully unsupervised PEGASUS model $\left(r_{\text {unsup }}\right)$. As can be seen, an untrained model indeed assigns much less likelihood to those examples, thus downweighting their influence on the

\begin{tabular}{|c|c|c|c|c|}
\hline$\ell$ & Model & Reddit TIFU & NEWSROOM & CNN/DailyMail \\
\hline \multirow[t]{2}{*}{$2^{7}$} & PEGA & $18.48 / 3.97 / 14$ & $25.59 / 1$ & $37.54 /$ \\
\hline & GENPET & $22.43 / \mathbf{5 . 5 5} / 17.27$ & $34.48 / 22.00 / 30.60$ & $38.91 / 16.97 / 26.65$ \\
\hline \multirow{2}{*}{$2^{5}$} & Pegasus & $18.76 / 3.97 / 14.36$ & $1.41 / 20.49$ & 31.8 \\
\hline & GENPET & $\mathbf{2 2 . 4 5} / 5.54 / \mathbf{1 7 . 3 2}$ & $33.89 / 21.26 / 30.02$ & $33.44 / 14.35 / 24.17$ \\
\hline
\end{tabular}

Table 5: R1/R2/RL scores with maximum output lengths of $2^{5}=32$ and $2^{7}=128$ given 10 training examples

final model. For example, the last text shown in Table 6 is more probable than $92 \%$ of all generated texts according to the trained model, compared to $24 \%$ for the untrained model. With unsupervised scoring, the first three examples shown are even completely removed from the training set for the final model as their rank is below the chosen threshold of $\tau=0.2$.

Variance To quantify the significance of performance improvements with GENPET over our two baselines, Pegasus and PegasusM, Table 7 shows the standard deviation of Rouge1/Rouge2/RougeL scores across the three different training sets for all tasks considered. 


\begin{tabular}{lcc}
\hline Text & $r_{\text {sup }}$ & $r_{\text {unsup }}$ \\
\hline Margaret Thatcher, [...] was one of the most visible, vocal and controversial leaders in the world. & 0.77 & 0.19 \\
Bruce Forsyth [...] was one of the most visible, vocal and controversial entertainers in the business. & 0.51 & 0.18 \\
[... ] Hawaii Five-O, a police drama that was one of the most visible, vocal and controversial of all-time. & 0.41 & 0.11 \\
Mongolia is one of the most visible, vocal and controversial countries in the world. & 0.81 & 0.32 \\
The state pension is one of the most visible, vocal and controversial of all-time. & 0.92 & 0.24 \\
\hline
\end{tabular}

Table 6: Texts generated by PEGASUS trained with individual patterns using GENPET on an XSum training set. Each of the five texts contains a phrase (highlighted in bold) from one specific training example. The right columns show the (normalized) rank of each output both with supervised scoring $\left(r_{\text {sup }}\right)$ and unsupervised scoring $\left(r_{\text {unsup }}\right)$. In these five examples, unsupervised scoring more effectively identifies the "parroted" phrase as not being a good fit for its new context.

\begin{tabular}{|c|c|c|c|c|}
\hline$|T|$ & Model & AESLC & Gigaword & XSum \\
\hline 10 & $\begin{array}{l}\text { PEGASUS } \\
\text { PEGASUS-M } \\
\text { GENPET }\end{array}$ & 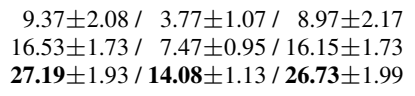 & $\begin{array}{l}25.18 \pm 0.77 / 9.24 \pm 0.41 / 22.80 \pm 0.61 \\
27.33 \pm 0.51 / 10.60 \pm 0.34 / 24.98 \pm 0.46 \\
\mathbf{3 0 . 9 3} \pm 0.15 / \mathbf{1 3 . 0 2} \pm 0.17 / \mathbf{2 8 . 4 9} \pm 0.17\end{array}$ & $\begin{array}{l}30.41 \pm 0.44 / \quad 9.57 \pm 0.27 / 23.26 \pm 0.29 \\
33.96 \pm 1.52 / 11.90 \pm 1.09 / 26.29 \pm 1.53 \\
\mathbf{3 5 . 8 8} \pm 1.42 / \mathbf{1 3 . 2 2} \pm 1.17 / \mathbf{2 8 . 2 4} \pm 1.50\end{array}$ \\
\hline 100 & $\begin{array}{l}\text { PEGASUS } \\
\text { PEGASUS-M } \\
\text { GENPET }\end{array}$ & $\begin{array}{l}23.22 \pm 0.29 / 10.24 \pm 0.46 / 22.43 \pm 0.28 \\
25.87 \pm 0.06 / 12.34 \pm 0.11 / 24.99 \pm 0.13 \\
\mathbf{2 9 . 9 7} \pm 0.39 / \mathbf{1 5 . 3 2} \pm 0.36 / \mathbf{2 9 . 2 6} \pm 0.54\end{array}$ & $\begin{array}{l}30.80 \pm 0.52 / 12.27 \pm 0.50 / 27.92 \pm 0.49 \\
31.38 \pm 0.05 / 12.65 \pm 0.19 / 28.33 \pm 0.12 \\
\mathbf{3 2 . 7 5} \pm 0.26 / \mathbf{1 3 . 9 8} \pm 0.09 / \mathbf{2 9 . 9 4} \pm 0.16\end{array}$ & $\begin{array}{l}40.23 \pm 0.10 / 16.68 \pm 0.10 / 31.90 \pm 0.06 \\
40.73 \pm 0.06 / 17.10 \pm 0.03 / 32.43 \pm 0.04 \\
\mathbf{4 1 . 7 1} \pm 0.06 / \mathbf{1 7 . 9 9} \pm 0.02 / \mathbf{3 3 . 4 6} \pm 0.08\end{array}$ \\
\hline$|T|$ & Model & Reddit TIFU & NEWSROOM & CNN/DailyMail \\
\hline 10 & $\begin{array}{l}\text { PEGASUS } \\
\text { PEGASUS-M } \\
\text { GENPET }\end{array}$ & $\begin{array}{ll}18.48 \pm 0.85 / & 3.97 \pm 0.26 / 14.08 \pm 0.41 \\
19.78 \pm 1.44 / & 4.50 \pm 0.39 / 15.16 \pm 0.84 \\
\mathbf{2 2 . 4 3} \pm 0.78 / & \mathbf{5 . 5 5} \pm 0.30 / \mathbf{1 7 . 2 7} \pm 0.30\end{array}$ & $\begin{array}{l}25.59 \pm 1.07 / 12.28 \pm 1.29 / 21.18 \pm 1.11 \\
29.91 \pm 0.29 / 16.73 \pm 0.37 / 25.70 \pm 0.26 \\
\mathbf{3 4 . 4 8} \pm 0.74 / \mathbf{2 2 . 0 0} \pm 0.70 / \mathbf{3 0 . 6 0} \pm 0.71\end{array}$ & $\begin{array}{l}37.54 \pm 0.39 / 15.84 \pm 0.27 / 25.18 \pm 0.27 \\
37.88 \pm 0.63 / 16.19 \pm 0.34 / 25.82 \pm 0.23 \\
\mathbf{3 8 . 9 1} \pm 0.56 / \mathbf{1 6 . 9 7} \pm 0.19 / \mathbf{2 6 . 6 5} \pm 0.14\end{array}$ \\
\hline 100 & $\begin{array}{l}\text { Pegasus } \\
\text { Pegasus-M } \\
\text { GENPet }\end{array}$ & $\begin{array}{ll}24.24 \pm 0.32 / & 6.28 \pm 0.01 / 18.72 \pm 0.27 \\
24.74 \pm 0.08 / & 6.40 \pm 0.05 / 19.10 \pm 0.01 \\
\mathbf{2 6 . 0 6} \pm 0.07 / & \mathbf{7 . 3 4} \pm 0.09 / \mathbf{2 0 . 3 4} \pm 0.12\end{array}$ & $\begin{array}{l}33.13 \pm 0.47 / 20.24 \pm 0.80 / 28.80 \pm 0.48 \\
34.79 \pm 0.55 / 21.60 \pm 0.74 / 30.37 \pm 0.54 \\
\mathbf{3 6 . 2 0} \pm 0.56 / \mathbf{2 3 . 5 1} \pm 0.69 / \mathbf{3 2 . 0 2} \pm 0.54\end{array}$ & $\begin{array}{l}39.64 \pm 0.13 / 16.94 \pm 0.16 / 26.79 \pm 0.18 \\
\mathbf{4 0 . 0 8} \pm 0.23 / 17.14 \pm 0.09 / 27.06 \pm 0.07 \\
40.02 \pm 0.22 / \mathbf{1 7 . 7 7} \pm 0.07 / \mathbf{2 7 . 7 9} \pm 0.05\end{array}$ \\
\hline
\end{tabular}

Table 7: Average R1/R2/RL scores and standard deviation $( \pm)$ for 10 and 100 training examples across three different (seed-dependent) training sets. 